\title{
Properties of the density-wave phase of a two-dimensional dipolar fermi gas
}

\author{
J. K. Block* and G. M. Bruun \\ Department of Physics and Astronomy, Aarhus University, DK-8000 Aarhus C, Denmark
}

(Dated: May 26, 2022)

\begin{abstract}
The rapid progress in the production and cooling of molecular gases indicates that experimental studies of quantum gases with a strong dipolar interaction is soon within reach. Dipolar gases are predicted to exhibit very rich physics including quantum liquid crystal phases such as density-waves as well as superfluid phases, both of which play an important role for our understanding of strongly correlated systems. Here, we investigate the zero temperature properties of the density-wave phase of a two-dimensional (2D) system of fermonic dipoles using a conserving Hartree-Fock theory. We calculate the amplitude of the density waves as a function of the dipole moment and orientation with respect to the $2 \mathrm{D}$ plane. The stripes give rise to a $1 \mathrm{D}$ Brillouin zone structure, and the corresponding quasiparticle spectrum is shown to have gapped as well as gapless regions around the Fermi surface. As a result, the system remains compressible in the density-wave phase, and it collapses for strong attraction. We show that the density-waves has clear signatures in the momentum distribution and in the momentum correlations. Both can be measured in time-of-flight experiments. Finally, we discuss how the striped phase can be realised with experimentally available systems.
\end{abstract}

PACS numbers: 03.75.Ss, 64.70.mf, 67.85.Lm, 68.65.Ac, 71.45.Lr

\section{INTRODUCTION}

The investigation of ultracold atomic gases has produced several breakthrough results in the last two decades ${ }^{1 / 2}$. Atomic gases are used to simulate many-body systems without the presence of disorder, intricate band structures etc., which significantly complicates the understanding of conventional condensed matter systems. One limitation is that the atom-atom interaction is typically short range and isotropic ( $s$-wave), whereas the order parameters in nature often exhibit richer $p$ - and $d$ wave symmetries. The impressive progress in the production of cold gases consisting of fermionic hetero-nuclear molecules with an electric dipole moment $\sqrt[3]{-9}$ promises to remove this limitation, since the dipole-dipole interaction is long-range and anisotropic with both repulsive and attractive parts 10 . The attractive head-to-tail part of the dipole-dipole interaction can lead to severe losses via chemical reactions, which however can be suppressed by orders of magnitude by confining the dipoles to low dimensional geometries ${ }^{11 \mid 12}$, or by using molecules which are chemically stable such as ${ }^{23} \mathrm{Na}^{40} \mathrm{~K}^{6 / 7}$ or ${ }^{40} \mathrm{~K}^{133} \mathrm{Cs}$. Dipolar gases are predicted to exhibit a wealth of new phases in 2D, including $p$-wave superfluids $13 \mid 14$ as well as quantum liquid crystals such as nematic ${ }^{15}$, density-wave (smectic) 16 and hexatic phases $24 \mid 25$. The presence of both superfluid and liquid crystal order occurs in several strongly correlated systems, and it plays a central role in the physics of the cuprate and pnictide superconductors $26 \mid 27$.

We analyse in this paper the zero temperature $(T=0)$ properties of the density-wave phase of a $2 \mathrm{D}$ gas of fermionic dipoles aligned by an external field. In this phase, the dipoles form density waves (stripes) in order to minimise the repulsive side-by-side part of the interaction. Several groups have predicted a $2 \mathrm{D}$ dipolar gas to form such a striped phase for large dipole moments 16 |22.
Partitioning momentum space into one dimensional Brillouin zones, we develop a conserving Hartree-Fock approximation (HFA) 29 , which is shown to recover previous results for the critical coupling strength for the onset of stripe formation. The resulting equations are solved self-consistently, and we calculate the amplitude of the stripes as a function of the dipole moment and its orientation with respect to the $2 \mathrm{D}$ plane. We then calculate the quasi-particle spectrum and show that the 1D Brillouin zone structure gives rise to a Fermi surface with gapless as well as gapped regions. As a result, the system remains compressible, and the formation of stripes does not stabilise the system against collapse for large dipole attraction. The presence of stripes is demonstrated to have clear signatures in the momentum distribution and to give rise to characteristic momentum correlations, both of which can be measured in time-of-flight experiments. Finally, we show how the effects described in this paper can be observed with experimentally available dipolar gases.

\section{SYSTEM}

We consider identical fermionic dipoles of mass $m$ moving in a $2 \mathrm{D}$ layer defining the $x y$-plane at $T=0$. The dipole moment $\mathbf{p}$ of the fermions is aligned forming the angle $\theta$ with respect to the normal of the layer ( $z$-axis) with its projection onto the planes defining the $x$-axis, see figure 1. We assume that the layer is formed by a deep 1D optical lattice so that the dipoles reside in the lowest harmonic oscillator level $\varphi(z)=\exp \left(-z^{2} / 2 w^{2}\right) \pi^{-1 / 4} w^{-1 / 2}$ in the z-direction with $w$ the width of the layer. We neglect any trapping potential in the $x y$-plane so that the transverse states are labelled by the momentum $\mathbf{k}=\left(k_{x}, k_{y}\right)$ (we take $\hbar=k_{B}=1$ ). 


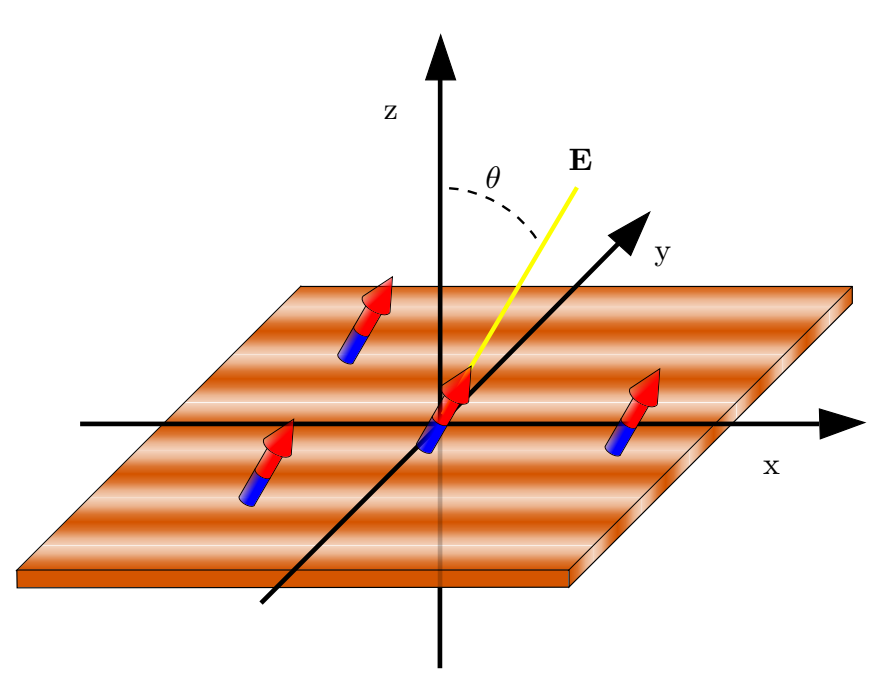

Figure 1. (colour online). The dipoles are confined in the $x y$-plane, and they are aligned by an external electrical field $\mathbf{E}$ forming the angle $\theta$ with respect to the $z$-axis. The density waves are along the $x$-axis which is defined by the projection of the field $\mathbf{E}$ onto the plane.

The Hamiltonian of the system is

$$
\hat{H}=\sum_{\mathbf{k}} \frac{k^{2}}{2 m} \hat{c}_{\mathbf{k}}^{\dagger} \hat{c}_{\mathbf{k}}+\frac{1}{2 A} \sum_{\mathbf{k}, \mathbf{k}^{\prime}, \mathbf{q}} V(\mathbf{q}) \hat{c}_{\mathbf{k}+\mathbf{q}}^{\dagger} \hat{c}_{\mathbf{k}^{\prime}-\mathbf{q}}^{\dagger} \hat{c}_{\mathbf{k}^{\prime}} \hat{c}_{\mathbf{k}}
$$

where $\hat{c}_{\mathbf{k}}$ removes a dipole with momentum $\mathbf{k}$. The interaction between two dipoles separated by $\mathbf{r}$ is $V_{3 \mathrm{D}}(\mathbf{r})=$ $D^{2}\left[1-3 \cos ^{2}\left(\theta_{r}\right)\right] / r^{3}$ where $\theta_{r}$ is the angle between $\mathbf{r}$ and the dipole moment $\mathbf{p}$, and $D^{2}=p^{2} / 4 \pi \varepsilon_{0}$ for electric dipoles. The effective interaction $V(\mathbf{q})$ in (1) is obtained by integrating the interaction $V_{3 \mathrm{D}}(\mathbf{r})$ over the Gaussians $\varphi(z)^{2}$, which yields 30

$$
V(\mathbf{q})=\pi D^{2}\left[\frac{8}{3 w \sqrt{2 \pi}} P_{2}(\cos \theta)-2 \xi(\theta, \varphi) F(q)\right] .
$$

Here, $P_{2}(x)=\left(3 x^{2}-1\right) / 2$ is the second Legendre polynomial, $F(q)=q \exp \left[(q w)^{2} / 2\right] \operatorname{erfc}(q w / \sqrt{2})$ and $\xi(\theta, \varphi)=$ $\cos (\theta)^{2}-\sin (\theta)^{2} \cos (\varphi)^{2}$. The constant term in equation (2) corresponds to a contact interaction which plays no role here, since we consider identical fermions. We characterise the strength of the interaction by the ratio of the typical interaction and kinetic energy,

$$
g=\frac{4 m D^{2} k_{F}^{0}}{3 \pi \hbar^{2}},
$$

where $k_{F}^{0}=\sqrt{4 \pi \rho_{0}}$ is defined from the areal density $\rho_{0}$. We likewise define the Fermi energy of a noninteracting system of the same density as $E_{F}^{0}=k_{F}^{0}{ }^{2} / 2 m$. For simplicity we only consider the limit $w \rightarrow 0$ corresponding to a strict $2 \mathrm{D}$ system. A non-zero value of $w$ leads to qualitatively the same physics with only a small shift in the critical coupling strength for stripe formation, as long as $k_{F}^{0} w \ll 1^{222}$.

\section{MEAN-FIELD THEORY}

The instability towards forming a striped phase is signalled by a zero frequency pole in the density-density response function at a given wave number $\mathbf{q}_{c}^{222}$. Close to the transition to the normal phase, the density modulation is dominated by the lowest Fourier components $\mathbf{q}_{c}$ and $-\mathbf{q}_{c}$, and we can write

$$
\rho(\mathbf{r})=\rho_{0}+\rho_{1} \cos \left(\mathbf{q}_{c} \cdot \mathbf{r}-u\right)
$$

with $A^{-1} \sum_{\mathbf{k}}\left\langle\hat{c}_{\mathbf{k}}^{\dagger} \hat{c}_{\mathbf{k}}\right\rangle=\rho_{0}$ and $A^{-1} \sum_{\mathbf{k}}\left\langle\hat{c}_{\mathbf{k}}^{\dagger} \hat{c}_{\mathbf{k}+\mathbf{q}_{c}}\right\rangle=$ $\rho_{1} \exp (i u) / 2$. Here, $u$ is the phase shift of the wave and $A$ is the area of the system. The lowest Fourier components $\mathbf{q}_{c}$ and $-\mathbf{q}_{c}$ also dominate deeper into the striped phase, and in the following we therefore neglect the contribution of higher harmonics to $\rho(\mathbf{r})$. Using Wick's theorem to expand the interaction part of the Hamiltonian (1), we construct a mean-field theory by including $\left\langle\hat{c}_{\mathbf{k}}^{\dagger} \hat{c}_{\mathbf{k}^{\prime}}\right\rangle$ for $\mathbf{k}=\mathbf{k}^{\prime}$ and $\mathbf{k}=\mathbf{k}^{\prime} \pm \mathbf{q}_{c}$ which yields the mean-field Hamiltonian

$$
\hat{H}_{\mathrm{MF}}=\sum_{\mathbf{k}} \varepsilon(\mathbf{k}) \hat{c}_{\mathbf{k}}^{\dagger} \hat{c}_{\mathbf{k}}+\sum_{\mathbf{k}}\left[\mathrm{h}(\mathbf{k}) \hat{c}_{\mathbf{k}+\mathbf{q}_{c}^{\dagger}} \hat{c}_{\mathbf{k}}+\text { h.c. }\right] .
$$

Here,

$$
\varepsilon(\mathbf{k})=\frac{k^{2}}{2 m}+\frac{1}{A} \sum_{\mathbf{k}^{\prime}}\left[V(0)-V\left(\mathbf{k}-\mathbf{k}^{\prime}\right)\right]\left\langle\hat{c}_{\mathbf{k}^{\prime}}^{\dagger} \hat{c}_{\mathbf{k}^{\prime}}\right\rangle
$$

is the Hartree-Fock single particle energy and

$$
\mathrm{h}(\mathbf{k})=\frac{1}{A} \sum_{\mathbf{k}^{\prime}}\left[V\left(\mathbf{q}_{c}\right)-V\left(\mathbf{k}-\mathbf{k}^{\prime}\right)\right]\left\langle\hat{c}_{\mathbf{k}^{\prime}}^{\dagger} \hat{c}_{\mathbf{k}^{\prime}+\mathbf{q}_{c}}\right\rangle .
$$

As usual, these parameters have to be determined selfconsistently. This is complicated significantly by the fact that $\mathrm{h}(\mathbf{k})$ is a function of $\mathbf{k}$, since it includes the exchange interaction $V\left(\mathbf{k}-\mathbf{k}^{\prime}\right)$. It is however crucial to include exchange, since it is known to lead to important effects such as the collapse of the system, a large deformation of the Fermi surface ${ }^{13 / 31}$, and a significant change in the critical coupling strength for the stripe instability $202|22| 32$. All these effects are recovered in our calculation as will be discussed below. For the self-consistent solution, we choose $u=0$ in (4) which corresponds to $\mathrm{h}(\mathbf{k})$ real.

\section{A. Band structure}

In the striped phase, the translational symmetry is spontaneously broken in the direction perpendicular to the stripes in analogy with a classical smectic liquid crysta 33 , while it is conserved in the direction along the stripes. Each dipole experiences the mean-field potential 
from the other dipoles which is periodic in the direction perpendicular to the stripes. It follows that a dipole with momentum $\mathbf{k}$ is coupled only to dipoles with momenta $\mathbf{k} \pm n \mathbf{q}_{c}$ with $n$ an integer. This allows us to think of $k$-space in terms of having a 1D Brillouin zone structure in the direction of $\mathbf{q}_{c}$ and an unrestricted $k$-space in the direction perpendicular to $\mathbf{q}_{c}$. We therefore partition the 2D $k$-space into slices of width $q_{c}$, by starting with the first Brillouin zone $B_{0}$ defined as the points $\mathbf{k}$ such that $-q_{c} / 2<\mathbf{k} \cdot \hat{\mathbf{q}}_{c} \leq q_{c} / 2$, where $\hat{\mathbf{q}}_{c}$ is the unit vector in the direction of $\mathbf{q}_{c}$. Then any $k$-space point $\mathbf{k}$ can be uniquely written as

$$
\mathbf{k}=\mathbf{k}^{\prime}+n \mathbf{q}_{c} \quad \text { where } \mathbf{k}^{\prime} \in B_{0} \text { and } n \in \mathbb{Z}
$$

The higher order zones are denoted by $B_{n}=\{\mathbf{k} \in$ $\left.\mathbb{R}^{2} \mid \exists \mathbf{k}^{\prime} \in B_{0}: \mathbf{k}=\mathbf{k}^{\prime}+n \mathbf{q}_{c}\right\}$, and the full $k$-space is the disjoint union of all $B_{n}$ 's. With this partitioning each $\mathbf{k}$ state only couples to itself and precisely one state in each of the two neighbouring Brillouin zones, and the mean-field Hamiltonian (5) can be written as a sum over Hamiltonians for each $\mathbf{k}$ in the first Brillouin zone: $\hat{H}_{\mathrm{MF}}=\sum_{\mathbf{k} \in B_{0}} \hat{\mathbf{c}}_{\mathbf{k}}^{\dagger} \mathbf{H}(\mathbf{k}) \hat{\mathbf{c}}_{\mathbf{k}}$. Here $\mathbf{H}(\mathbf{k})$ is a tridiagonal matrix describing the coupling between states with momenta $\mathbf{k}+n \mathbf{q}_{c}$ and $\hat{\mathbf{c}}_{\mathbf{k}}^{\dagger}=\left(\ldots, \hat{c}_{\mathbf{k}-n \mathbf{q}_{c}}^{\dagger}, \ldots, \hat{c}_{\mathbf{k}}^{\dagger}, \hat{c}_{\mathbf{k}+\mathbf{q}_{c}}^{\dagger}, \ldots, \hat{c}_{\mathbf{k}+n \mathbf{q}_{c}}^{\dagger}, \ldots\right)$. So the mean-field Hamiltonian can be diagonalised for each $\mathbf{k}$ in $B_{0}$ separately. Note however that the self-consistent averages in (6)-(7) are determined by summing over all $\mathbf{k}$, thereby coupling different k's.

For each $\mathbf{k}$ in the first Brillouin zone, we diagonalise the Hamiltonian $\mathbf{H}(\mathbf{k})$ by introducing the quasi-particle operator $\hat{\gamma}_{\mathbf{k}}=\mathbf{U}(\mathbf{k})^{\dagger} \hat{\mathbf{c}}_{\mathbf{k}}$ so that $\mathbf{U}(\mathbf{k})^{\dagger} \mathbf{H}(\mathbf{k}) \mathbf{U}(\mathbf{k})=\mathbf{D}(\mathbf{k})$ is a diagonal matrix with quasi-particle energies $E_{i}(\mathbf{k})$ on the diagonal. The thermal average of the new operators for $\mathbf{k}, \mathbf{k}^{\prime} \in B_{0}$ is given by $\left\langle\hat{\gamma}_{\mathbf{k}, i}^{\dagger} \hat{\gamma}_{\mathbf{k}^{\prime}, j}\right\rangle=\delta_{\mathbf{k}, \mathbf{k}^{\prime}} \delta_{i, j} f\left[E_{i}(\mathbf{k})\right]$, where $f(x)=[\exp \beta(x-\mu)+1]^{-1}$ is the Fermi-Dirac distribution and $\mu$ is the chemical potential. We can then calculate the Hartree-Fock energies and the order parameter selfconsistently from the diagonal Hamiltonian. The chemical potential is determined by keeping the density of dipoles fixed.

With the geometry illustrated in Fig. 1, the stripes are parallel to the $k_{x}$-axis, since this minimises the repulsion between the dipoles. Thus, the vector $\mathbf{q}_{c}$ is parallel to the $k_{y}$-axis corresponding to $\varphi_{c}=\pi / 2$, where $\varphi$ is the azimuthal angle between a vector in $\mathbf{k}$-space and the $k_{x}$-axis. We furthermore choose $q_{c}=2 k_{F}\left(\varphi_{c}, \theta, g\right)$ since we expect this to lead to the lowest critical coupling strength for stripe formation, as the density wave can then be formed by particle-hole excitations around the Fermi surface with no cost in kinetic energy. Here $k_{F}(\varphi, \theta, g)$ is the length of the Fermi vector in the normal phase for interaction strength $g$ and dipole tilting $\theta$. It depends on the angle $\varphi$, since the Fermi surface is deformed by the dipole-dipole interaction forming an elliptical shape, see Fig. 4. We calculate the deformation using the variational method based on Hartree Fock theory described in 13 .

\section{B. Three band theory}

To proceed, we reduce the numerical complexity by truncating the matrix $\mathbf{H}(\mathbf{k})$ which is to be diagonalized at each point in the first Brillouin zone. As shown in the appendix A, the condition for the stripe instability obtained from calculating the density-density response function in the conserving HFA involves for $T=0$ momenta only in the three lowest Brillouin zones $B_{n}$ with $n=-1,0,1$. Thus, we include these three Brillouin zones in our calculations whereas higher energy zones are neglected. In this way we recover the instability line obtained in Ref.22. Higher Brillouin zones contribute in the striped phase or at non-zero temperature, but as long as $T \ll \varepsilon_{F}$ and $\mathrm{h}(\mathbf{k}) \ll \varepsilon_{F}$ their contribution is negligible. We shall later demonstrate numerically that including the lowest three zones only is an excellent approximation for the parameters chosen.

The mean-field Hamiltonian for a given $\mathbf{k} \in B_{0}$ is then

$$
\mathbf{H}(\mathbf{k})=\left[\begin{array}{ccc}
\varepsilon_{\mathbf{k}-\mathbf{q}_{c}} & \mathrm{~h}_{\mathbf{k}-\mathbf{q}_{c}}^{*} & 0 \\
\mathrm{~h}_{\mathbf{k}-\mathbf{q}_{c}} & \varepsilon_{\mathbf{k}} & \mathrm{h}_{\mathbf{k}}^{*} \\
0 & \mathrm{~h}_{\mathbf{k}} & \varepsilon_{\mathbf{k}+\mathbf{q}_{c}}
\end{array}\right]
$$

and the three quasi particle energy bands $E_{\mathbf{k}, 1} \leq E_{\mathbf{k}, 2} \leq$ $E_{\mathbf{k}, 3}$ are the eigenvalues of the matrix $\mathbf{H}(\mathbf{k})$.

The self-consistent equations (6) for the Hartree Fock energy and (7) for the order parameter read in terms of the new single particle eigenstates

$$
\begin{aligned}
\varepsilon(\mathbf{k})= & \frac{k^{2}}{2 m}+\frac{1}{A} \sum_{\mathbf{k}^{\prime} \in B_{0}} \sum_{n=-1}^{1}\left[V(0)-V\left(\mathbf{k}-\mathbf{k}^{\prime}-n \mathbf{q}_{c}\right)\right] \times \sum_{l=1}^{3}\left|U\left(\mathbf{k}^{\prime}\right)_{n+2, l}\right|^{2} f\left(E_{\mathbf{k}^{\prime}, l}\right) \\
\mathrm{h}(\mathbf{k})= & \frac{1}{A} \sum_{\mathbf{k}^{\prime} \in B_{0}}\left\{\left[V\left(\mathbf{q}_{c}\right)-V\left(\mathbf{k}-\mathbf{k}^{\prime}+\mathbf{q}_{c}\right)\right] \times \sum_{l=1}^{3} U\left(\mathbf{k}^{\prime}\right)_{1, l}^{*} U\left(\mathbf{k}^{\prime}\right)_{2, l} f\left(E_{\mathbf{k}^{\prime}, l}\right)\right. \\
& \left.\left.+\left[V\left(\mathbf{q}_{c}\right)-V\left(\mathbf{k}-\mathbf{k}^{\prime}\right)\right] \times \sum_{l=1}^{3} U\left(\mathbf{k}^{\prime}\right)_{2, l}^{*} U\left(\mathbf{k}^{\prime}\right)\right)_{3, l} f\left(E_{\mathbf{k}^{\prime}, l}\right)\right\}
\end{aligned}
$$


while the Fourier components of the density read

$$
\rho_{0}=\frac{1}{A} \sum_{\mathbf{k} \in B_{0}} \sum_{l=1}^{3} f\left(E_{\mathbf{k}, l}\right), \quad \text { and } \quad \rho_{1}=\frac{2}{A} \sum_{\mathbf{k} \in B_{0}} \sum_{l=1}^{3}\left[U(\mathbf{k})_{1, l}^{*} U(\mathbf{k})_{2, l}+U(\mathbf{k})_{2, l}^{*} U(\mathbf{k})_{3, l}\right] f\left(E_{\mathbf{k}, l}\right) .
$$

The equations (9)-(12) are solved self-consistently by discretizing $B_{0}$ using a rectangular grid including states up to $\pm 1.1 k_{F}(\varphi=0, g, \theta)$ in the $k_{x}$-direction. $B_{0}$ is defined as being infinite in the $k_{x}$ direction, but there is no coupling in the direction perpendicular to $\mathbf{q}_{c}$, so the states with $k_{x}$ outside the Fermi surface of the normal phase are not occupied. The grid is $n_{k_{x}} \times n_{k_{y}}=101 \times 161$ points with an increased density of points near the edges. The iteration procedure is as follows: For each $\mathbf{k}$-point the $3 \times 3$ matrix $\mathbf{U}(\mathbf{k})$ and the eigenenergies $E_{i}(\mathbf{k})$ are formed by diagonalization of $\mathbf{H}(\mathbf{k})$ computed using the current estimates to $\mathrm{h}$ and $\varepsilon$. Then $\mu$ is calculated such that the density is constant and finally the new estimates to $\rho_{1}, \mathrm{~h}$, and $\varepsilon$ are calculated from $(10)-(12)$. The iteration is terminated when the absolute change in $\rho_{1}$ is less than $10^{-6}$, while the maximum absolute change in any $\mathbf{k}$-point for $\mathrm{h}$ and $\varepsilon$ is less than $10^{-3} E_{F}^{0}$ and $5 \cdot 10^{-3} E_{F}^{0}$, respectively.

\section{RESULTS}

We now discuss the main results of our numerical calculations yielding self-consistent solutions to (9)-(11).

\section{A. Stripe amplitude}

Figure 2 shows the amplitude of the density wave $\rho_{1} / \rho_{0}$, which we take to be the order parameter of the striped phase, as a function of the coupling strength and alignment angle $\theta$. We clearly see the onset of stripe order beyond a critical coupling strength which depends on the angle. Note that the transition to the broken symmetry phase is not completely sharp since the discretisation of $k$-space in the numerical calculations corresponds to finite size effects, which result in a smooth crossover between the normal and the striped phase. Taking this small effect into account, the critical coupling strength for the stripe instability obtained here agrees well with the previous result based on linear response theory ${ }^{22}$, which is indicated by a white line in Fig. 2. This confirms the consistency of our approach and accuracy of the numerics of the present paper. We see that the order parameter $\rho_{1} / \rho_{0}$ quickly increases with increasing coupling strength resulting in significant density modulations in the striped phase.

For large tilting angles $\theta$ of the dipoles, Hartree-Fock theory predicts that the homogenous state is unstable against density collapse for strong coupling 13 . This instability is also predicted using a different theoretical

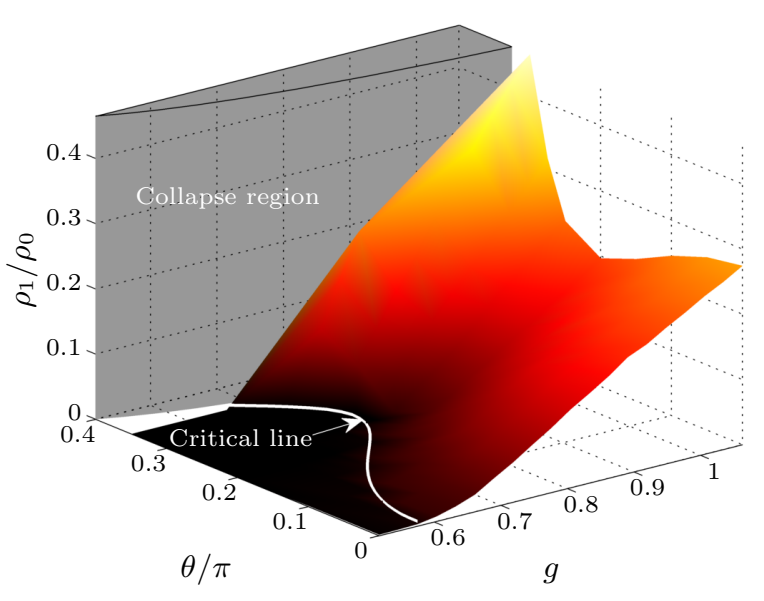

Figure 2. (colour online) The amplitude $\rho_{1} / \rho_{0}$ of the density wave as a function of the coupling strength $g$ and tilt angle $\theta$. The white line is the critical line for stripe formation obtained from linear response ${ }^{22}$, while the shaded region depicts the collapse region $13 \mid 17$.

approach obtaining almost the same critical coupling strength for collapse 21 . Since a broken symmetry in general leads to a gap in the spectrum thereby making the system less compressible, an interesting question is whether the striped phase stabilises the gas against this collapse. However, we do not find any numerical evidence of such a stabilising effect. On the contrary, our numerical calculations do not converge in the region where a homogeneous phase is predicted to collapse ${ }^{13 \mid 17}$, which is depicted by a shaded region on Fig. 2. This indicates that stripe order does not stabilise the systems against collapse. We speculate that the reason is that the systems remains gapless in certain regions of the Fermi surface in the striped phase, as we shall discuss in detail below.

In Fig. 3, we plot the stripe order parameter $\rho_{1} / \rho_{0}$ as a function of $g$ for various tilt angles $\theta$. These curves correspond to cuts along constant $\theta$ in Fig. 22. They clearly illustrate that apart from finite size effects, the critical coupling strength for the onset of pairing agrees well with what is obtained from a linear response ${ }^{22}$. An interesting effect is that the stripe amplitude increases faster for larger angles $\theta$, where the interaction is increasingly anisotropic and the system approaches the collapse instability. 


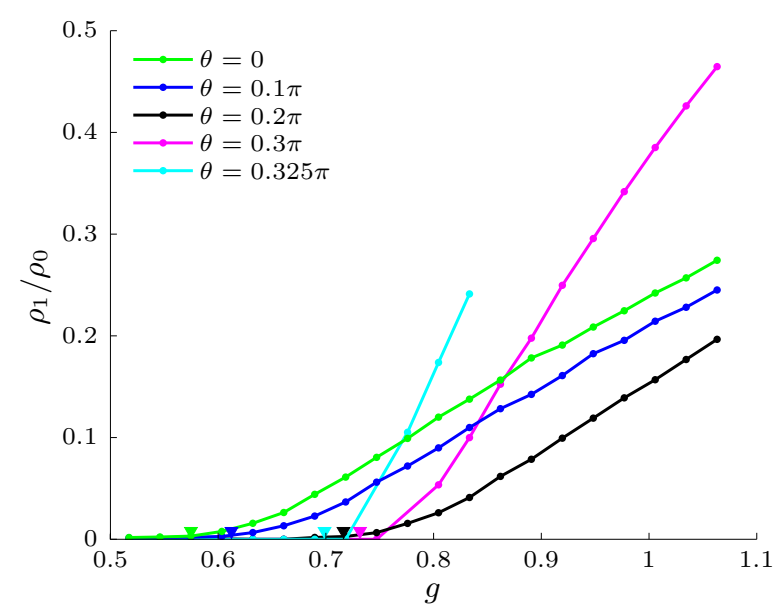

Figure 3. (colour online) $\rho_{1} / \rho_{0}$ as a function of $g$ for various tilt angles $\theta$. The critical value for stripe formation ${ }^{22}$ for each angle is marked by a triangle $\nabla$ of the corresponding color.

\section{B. Momentum distribution}

The momentum distribution $\left\langle\hat{c}_{\mathbf{k}}^{\dagger} \hat{c}_{\mathbf{k}}\right\rangle$ of the system can be measured in a time-of-flight (TOF) experiment, and we now analyse how this can be used to detect the striped phase. In Fig. 4, we plot $\left\langle\hat{c}_{\mathbf{k}}^{\dagger} \hat{c}_{\mathbf{k}}\right\rangle$ for $g=1.01$ and $\theta=0.3 \pi$ which corresponds to a fairly large stripe amplitude $\rho_{1} / \rho_{0}=0.385$. First, we notice that the momentum distribution is strongly anisotropic in agreement with what is found for the normal phase ${ }^{13131}$. We plot in Fig. 4 an elliptical approximation for the Fermi sea as calculated from a variational Hartree Fock theory for the normal phase, as described in 13 . We see that the Fermi sea for the striped phase has almost the same underlying elliptical shape. To illustrate the significant Fermi surface deformation, we also plot the circular Fermi sea of a noninteracting system with the same density. In addition to the elliptical shape of the Fermi sea, the striped phase is characterised by a smearing out of the momentum distribution in the regions located around the edge of the Fermi sea with $\varphi \simeq \pm \pi / 2$, i.e. $\mathbf{k} \simeq \pm \mathbf{q}_{c} / 2$. This is because the states with momenta $\mathbf{k}$ and $\mathbf{k} \mp \mathbf{q}_{c}$ are nearly degenerate in these regions where the Fermi surface is near the edge of the first Brillouin zones. The resulting strong mixing of the momentum states means that the quasiparticles do not have a well-defined momentum. Note that these regions are enlarged due to the underlying elliptical shape of the Fermi sea creating a "nesting" effect in analogy with lattice systems. It follows from this nesting that the stripe order is enhanced by the elliptical shape of the Fermi sea. In total, Fig. 4 clearly demonstrates that the striped phase can be detected in a TOF experiment by the characteristic shape of its Fermi sea.

Finally, Fig. 4 shows that the population in the Brillouin zones $B_{n}$ with $n= \pm 1$ is very small. This confirms that the three band approximation is accurate in the striped phase for the parameters used.

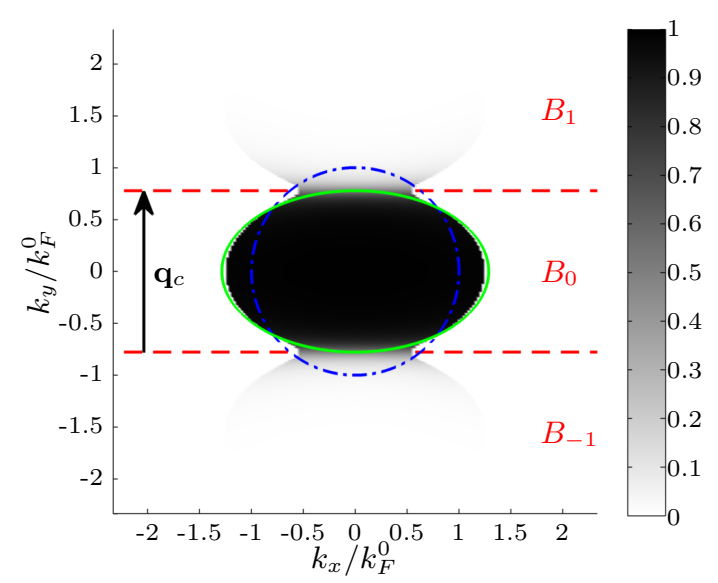

Figure 4. (colour online) The momentum distribution $\left\langle\hat{c}_{\mathbf{k}}^{\dagger} \hat{c}_{\mathbf{k}}\right\rangle$ in the striped phase for $g=1.01, \theta=0.3 \pi$ where $\rho_{1} / \rho_{0}=$ 0.385 , plotted in the three first Brillouin zones $B_{-1}, B_{0}$, and $B_{1}$. The elliptical shape of the underlying Fermi sea (solid green) of the homogenous phase and the circular Fermi sea (dashed blue) for a non-interacting system are also shown.

\section{Quasi-particle energies}

As we discussed, stripe order mixes states with momenta differing by $\mathbf{q}_{c}$ giving rise to large effects in the regions around $\mathbf{k} \simeq \pm \mathbf{q}_{c} / 2$. To examine this effect further, we plot in Fig. 5 the quasiparticle energies for the lowest two bands obtained from diagonalising the matrix $\mathbf{H}(\mathbf{k})$ for $g=1.01, \theta=0.3 \pi$ giving $\rho_{1} / \rho_{0}=0.385$. As expected, we see that the stripe order gives rise to a gap opening up at the Fermi surface in the regions around $\mathbf{k} \simeq \pm \mathbf{q}_{c} / 2$. The system however remains gapless in the other regions of the Fermi surface where the quasiparticle energies are perturbed only slightly from their normal phase values. This is illustrated further in Fig. 6 where we plot the quasiparticle energies along cuts defined by $k_{x}=0$ and $k_{y}=0$. One clearly sees the gap at the Fermi surface for $k_{x}=0$ whereas there is no gap for $k_{y}=0$. This explains why the system remains compressible and the stripe order does not stabilise the system significantly against collapse. It furthermore opens up the intriguing possibility of forming stripe and superfluid order simultaneously: While Cooper pairing is suppressed in the gapped regions around $\mathbf{k} \simeq \pm \mathbf{q}_{c} / 2$, particles around the gapless regions Fermi surface can still form Cooper pairs. Such a phase with both superfluid and density order is a supersolid, and its experimental realisation would be a major result, since it has not been unambiguously observed despite decades of intense research $\underline{34}$ 39. It also demonstratres that it is very promising to use dipolar gases to investigate the interplay between quantum liquid crystal phases such a stripes, and superfluid pairing, 


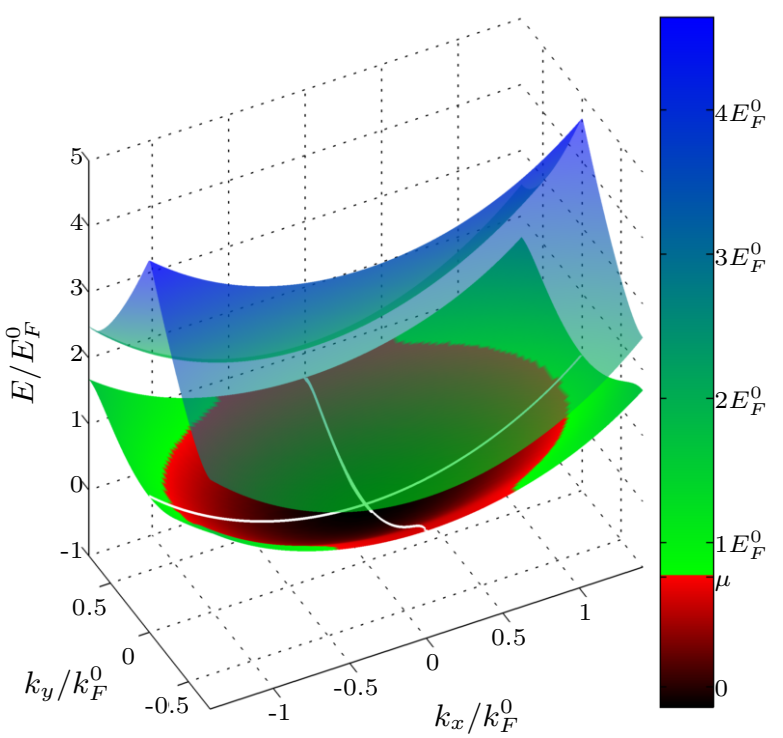

Figure 5. (colour online) The two lowest energy bands for $g=$ 1.01 and $\theta=0.3 \pi$. Energies below the chemical potential are colored red, energies above the chemical potential are colored green going to blue. The white lines indicate the cuts along $k_{x}=0$ and $k_{y}=0$ which are shown in Fig. 6 .

which is a central topic in the physics of strongly correlated systems including cuprate and pnictide superconductors 2622 .
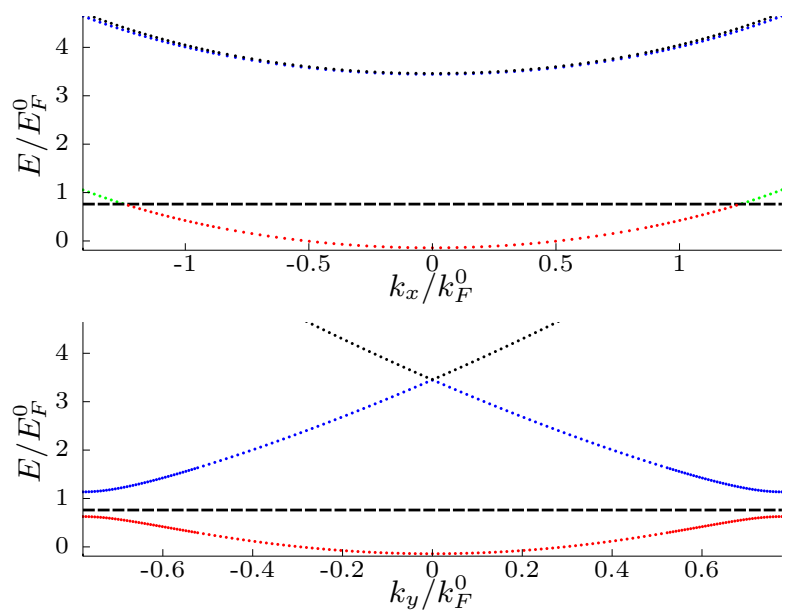

Figure 6. (colour online) The two lowest energy bands and the lower part of the third band along $k_{y}=0$ (top) and $k_{x}=0$ (bottom) in the first Brillouin zone. Occupied states in the lowest band are indicated by red dots, the chemical potential is indicated by a dashed line, while unoccupied states in the first, second, and third bands are indicated by green, blue and black dots respectively.

\section{Momentum correlations}

TOF experiments can also be used to measure correlation functions in quantum gases. Indeed, pair correlations 40 , bosonic bunching 4 , fermionic antibunching $\stackrel{42}{ }$, and the Mott-superfluid ${ }^{43}$ has been measured with this technique. We now demonstrate how TOF experiments can be used to detect the formation of stripes.

In TOF experiments, the density-density correlation function $\left\langle\rho(\mathbf{r}) \rho\left(\mathbf{r}^{\prime}\right)\right\rangle$ at points $\mathbf{r}$ and $\mathbf{r}^{\prime}$ can be measured after the trap has been switched off and the gas has been allowed to expand for a time $t$. Assuming free expansion, this corresponds to measuring the momentum correlation function $\left\langle n_{\mathbf{k}} n_{\mathbf{k}^{\prime}}\right\rangle$ before expansion with $\mathbf{k}=m \mathbf{r} / t$. We therefore analyse the correlation function

$$
\mathcal{C}\left(\mathbf{k}, \mathbf{k}^{\prime}\right)=\left\langle n_{\mathbf{k}} n_{\mathbf{k}^{\prime}}\right\rangle-\left\langle n_{\mathbf{k}}\right\rangle\left\langle n_{\mathbf{k}^{\prime}}\right\rangle=-\left\langle\hat{c}_{\mathbf{k}}^{\dagger} \hat{c}_{\mathbf{k}^{\prime}}\right\rangle\left\langle\hat{c}_{\mathbf{k}^{\prime}}^{\dagger} \hat{c}_{\mathbf{k}}\right\rangle,
$$

where we have used mean-field theory in the second equality and assumed $\mathbf{k} \neq \mathbf{k}^{\prime} . \mathcal{C}\left(\mathbf{k}, \mathbf{k}^{\prime}\right)$ is nonzero in the striped phase for $\mathbf{k}^{\prime}=\mathbf{k} \pm \mathbf{q}_{c}$, and taking $\mathbf{k}^{\prime}=\mathbf{k}+\mathbf{q}_{c}$ we obtain

$$
\mathcal{C}\left(\mathbf{k}, \mathbf{k}+\mathbf{q}_{c}\right)= \begin{cases}0 & \text { for } \mathbf{k} \in B_{1} \\ -\left|\sum_{l=1}^{3} U(\mathbf{k})_{1, l}^{*} U(\mathbf{k})_{2, l} f\left(E_{\mathbf{k}, l}\right)\right|^{2} & \text { for } \mathbf{k} \in B_{0} \\ -\left|\sum_{l=1}^{3} U\left(\mathbf{k}+\mathbf{q}_{c}\right)_{2, l}^{*} U\left(\mathbf{k}+\mathbf{q}_{c}\right)_{3, l} f\left(E_{\mathbf{k}+\mathbf{q}_{c}, l}\right)\right|^{2} & \text { for } \mathbf{k} \in B_{-1}\end{cases}
$$

In Fig. 7. we plot $\mathcal{C}\left(\mathbf{k}, \mathbf{k}+\mathbf{q}_{c}\right)$ as a function of $\mathbf{k}$ for $\quad g=1.01, \theta=0.3 \pi$. 


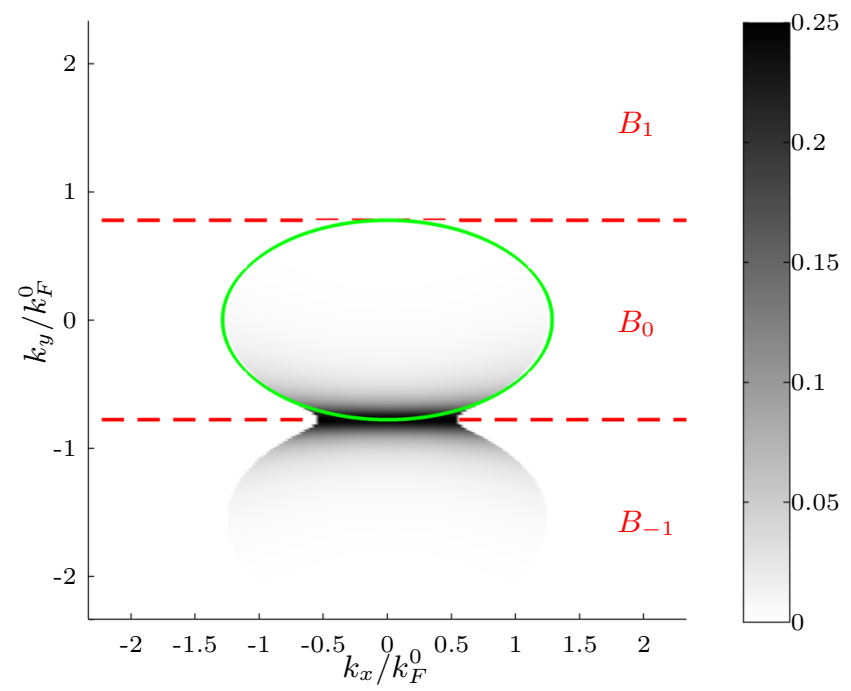

Figure 7. (color online) The correlation function $\mathcal{C}\left(\mathbf{k}, \mathbf{k}+\mathbf{q}_{c}\right)$ for $g=1.01, \theta=0.3 \pi$ in the first three Brillouin zones. It has a peak value of $1 / 4$ at $\mathbf{k}=-\mathbf{q}_{c} / 2$. Also shown is the Fermi surface for a homogenous phase with same tilting angle and interaction strength.

We clearly see a peak around $\mathbf{k}=-\mathbf{q}_{c} / 2$, where the nearly degenerate states on opposite sites of the Fermi ellipse strongly mix. Right at the Fermi surface for $k_{x}=0$, the states are fully mixed giving the maximum value $\left|\mathcal{C}\left(\mathbf{k}, \mathbf{k}+\mathbf{q}_{c}\right)\right|^{2}=1 / 4$. Note that $\mathcal{C}\left(\mathbf{k}, \mathbf{k}-\mathbf{q}_{c}\right)$ is the same as (14) mirrored around the $k_{x}$-axis. We conclude that stripe order can be detected by the presence of characteristic peaks in the density-density correlation function measured in a TOF experiment.

It should be noted that the dipolar interaction is long ranged compared to the usual atom-atom interaction, and it is therefore not obvious that it can be neglected during free expansion. However, we expect that the smoking gun features of the striped phase are robust toward interaction effects during TOF. In particular, the peak in the correlation function shown in Fig. 7 will survive interaction effects, since it is an inherent feature of the density wave. It might be distorted during expansion due to interactions, but it will not disappear. Also, it was found in Ref ${ }^{44}$ that for pancake shaped traps, which is the geometry we consider, dipolar interactions only have a small effect on the momentum distribution in TOF experiments. Even though they only analysed the case when the dipoles are perpendicular to the pancake, this indicates that interaction effects are small under TOF experiments for the system we consider.

\section{DISCUSSION}

Since stripe formation occurs for fairly strong coupling, one cannot expect in general the mean-field approach used in this paper to be quantitatively accurate. From the $1 \mathrm{D}$ character of the stripes, we do indeed expect fluctuations away from the mean-field result to be significant. These fluctuations will suppress stripe order and therefore increase the critical coupling strength for stripe formation. It is difficult to give a precise estimate of the accuracy of the mean-field approach. From the Ginzburg or Brout criterion, we expect the size of fluctuations to be determined by $h(k) / \varepsilon_{F}$.

One can also compare our prediction for the critical coupling strength with those obtained using different theoretical approaches. Calculations of the divergence of the density-density response function using the RPA give a critical coupling strength of $g \simeq 0.26$ for dipoles oriented perpendicular to the plane, $\theta=0$ 16/17. Including exchange correlations to form a conserving Hartree-Fock approximation increases the critical coupling strength to about $g=0.5 \sqrt{20122132}$ which is equivalent to the approach in the present paper. When the response function is calculated introducing a local field factor using the so called STLS scheme, one obtains $g=2.6$ for the critical coupling strength 21 . This approach does include correlations beyond Hartree-Fock, but at the same time it neglects the non-local nature of the correlations. For the special case of the dipoles perpendicular to the plane, fixed-node Monte-Carlo calculations indicate that the striped phase is preceded by a triangular Wigner crystal at $g=10.6 \pm 1.328$. However, these calculations use variational wave functions both for the normal and the striped phase with less than 100 particles, and the resulting energies of the two phases differ by less than $1 \%$. Thus, it is not clear how robust these results are to finite size effects and to improvements in the wave functions. Finally, a variational method based on wave functions for the 2D electron gas yields $g=11.9 \pm 1.7$ for the critical coupling strength of the Wigner crysta ${ }^{45}$, which is close to the diffusion Monte Carlo result, but to our knowledge this method has not been used to look for the density wave instability. In total, the large discrepancies between the different theoretical predictions show that the striped phase is a strongly correlated phenomenon. It is unfortunately not straightforward to rank the accuracy of the different results approaches, and the problem therefore calls for an experimental investigation. We emphasize that the analysis presented here based on the mean-field approach is the first one to describe the broken symmetry striped phase. Also, the mean-field results presented here such as the smoking gun features of the striped phase in the momentum distribution, must be expected to be qualitatively correct.

The striped phase spontaneously breaks translational symmetry, and it is therefore a quantum analogue of the classical smectic phases 33 . Quantum nematic and smectic phases play a significant role for many interesting electronic materials which have been discovered in the last couple of decades 2627 . Contrary to the electron systems, which are plagued by impurities, intricate band structures, lattice defects and distortions, that complicate a systematic analysis, ultracold dipolar gases are extremely 
clean and in addition experimentally very flexible. They therefore provide a great opportunity to investigate the formation of a smectic phase in a controlled and pure setting. In this paper, we approach these phases from the weak-coupling perspective, where the phases arise from the successive breaking of symmetries of an underlying Fermi surface ${ }^{46}$.

\section{EXPERIMENTAL CONSIDERATIONS}

The interaction strength depends strongly on the type of molecule used in an experiment, since it scales with $g \propto m p^{2} \sqrt{n_{2 \mathrm{D}}}$. As an example, consider the chemically stable ${ }^{23} \mathrm{Na}^{40} \mathrm{~K}$. To give a conservative estimate of experimentally realistic values, we refer to Refs. 3 and 11, where the JILA group reports a maximum value of the induced dipole moment $p / p_{0}$ of about 0.4 for the chemically unstable ${ }^{40} \mathrm{~K}^{87} \mathrm{Rb}$, and a maximum density of $3.4 \cdot 10^{7} \mathrm{~cm}^{-2}$ in a pancake geometry. If the same values are used for ${ }^{23} \mathrm{Na}^{40} \mathrm{~K}$, it corresponds to $g \simeq 1.0$ which is well within the striped phase as calculated in mean-field theory presented here. Similar parameters for the other chemically stable molecule ${ }^{40} \mathrm{~K}^{133} \mathrm{Cs}$ gives $g=1.3$, and taking $p=p_{0}$ yields $g=8.4$. Provided one can overcome difficulties related to the fact that ${ }^{6} \mathrm{Li}^{133} \mathrm{Cs}$ is chemically reactive by using the $2 \mathrm{D}$ geometry, the large permanent dipole moment $p_{0}=5.5$ Debye of this molecule means that one can even achieve the very high coupling strength $g \simeq 55$ for the same density. These very different values illustrate the quadratic dependence of the coupling strength on the dipole moment, which means that experiments likely will be able to probe a large region of the phase diagram. One should therefore be able to investigate the critical value for stripe formation, which is presently not settled theoretically as discussed above.

An interesting consideration is the effects of temperature. A finite and small temperature broadens the momentum distribution leading to less sharp signatures. In the strict $2 \mathrm{D}$ limit, no true long range order exists at non-zero temperature, and the phase transition to the homogenous phase is of the Berezinskii-Kosterlitz-Thouless (BKT) type ${ }^{47 / 48}$. For increasing temperatures, defects in the form of insertion and disappearance of stripes will proliferate eventually melting the stripes. In the strongly interacting limit we expect the BKT temperature to be proportional to the density, however the constant of proportionality has yet to be calculated. This will be explored in future work.

\section{CONCLUSIONS}

We studied the $T=0$ properties of the striped phase of a $2 \mathrm{D}$ system of fermionic dipoles aligned by an external field. A Hartree-Fock theory was developed, which was shown to recover previous results for the critical coupling strength for stripe formation. The amplitude of the stripes was calculated as a function of the dipole moment and orientation, and the quasiparticle spectrum of the striped phase was shown to exhibit a 1D Brillouin zone structure with gapped as well as gapless regions around the Fermi surface. The system therefore remains compressible in the striped phase, and it collapses for essentially the same dipole strength as in the normal phase. We showed that the striped phase has clear signatures in the momentum distribution and in the momentum correlations, which can both be measured in TOF experiments. Finally, we discussed how the striped phase can be realised with experimentally relevant molecules.

\section{ACKNOWLEDGMENTS}

We are grateful to Nikolaj Zinner for discussions and to the Centre for Scientific Computing in Aarhus for computation time. GMB would like to acknowledge the support of the Carlsberg Foundation via grant 2011010264 and the Villum Foundation via grant VKR023163.

\section{Appendix A: $k$-space in the conserving Hartree-Fock approximation}

To argue for the truncation of $k$-space, we examine the calculation of the static density-density response function $\chi$. The divergence of $\chi(\mathbf{q}, \omega=0)$ signifies the instability of the system towards forming density waves with wave vector $\mathbf{q}^{16 / 17}$ and thus marks the boundary of the DW phase. The self consistent mean-field theory employed in this study is an extension of the conserving ${ }^{29}$ HFA to the density-density response function as calculated in $20|22| 32$. So examining the latter approach gives an indication of which $k$-states are relevant in the vicinity of the phase transition. As shown in 22 , the internal Matsubara frequencies in the exchange plus direct interaction approximation to $\chi$ only appear in the particle-hole propagator $\Pi(k, q)=G(k+q) G(k)$, where $k=\left(\mathbf{k}, i k_{n}\right)$ is the $2+1$ momentum and $G$ is the fully dressed single particle Greens function. The Matsubara frequency sum is trivial so

$$
\begin{aligned}
& \sum_{k} \Pi(k, q)=\sum_{\mathbf{k}} \frac{f_{\mathbf{k}}-f_{\mathbf{k}+\mathbf{q}}}{i q_{n}+\varepsilon_{\mathbf{k}}-\varepsilon_{\mathbf{k}+\mathbf{q}}} \\
& =\sum_{\mathbf{k}}\left(\frac{f_{\mathbf{k}}}{i q_{n}+\varepsilon_{\mathbf{k}}-\varepsilon_{\mathbf{k}+\mathbf{q}}}+\frac{f_{\mathbf{k}}}{-i q_{n}+\varepsilon_{\mathbf{k}}-\varepsilon_{\mathbf{k}-\mathbf{q}}}\right)
\end{aligned}
$$

where $\varepsilon_{\mathbf{k}}$ is the Hartree Fock single particle energy as given by (6). Here we can see that particle-hole propagator is given exactly by the coupling between the occupied states of the lowest band $\varepsilon_{\mathbf{k}}$ to the two bands $\varepsilon_{\mathbf{k} \pm \mathbf{q}}$ which is captured by the three band model described in section IIIB. 
* jkblock@phys.au.dk

1 I. Bloch, J. Dalibard, and W. Zwerger, Reviews of Modern Physics 80, 885 (2008).

${ }^{2}$ S. Giorgini, L. L. Pitaevskii, and S. Stringari, Reviews of Modern Physics 80, 1215 (2008)

3 K.-K. Ni, S. Ospelkaus, D. Wang, G. Quéméner, B. Neyenhuis, M. H. G. de Miranda, J. L. Bohn, J. Ye, and D. S. Jin, Nature 464, 1324 (2010).

4 B. Deh, W. Gunton, B. G. Klappauf, Z. Li, M. Semczuk, J. Van Dongen, and K. W. Madison, Physical Review A 82, 020701 (2010)

${ }^{5}$ M.-S. Heo, T. T. Wang, C. A. Christensen, T. M. Rvachov, D. A. Cotta, J.-H. Choi, Y.-R. Lee, and W. Ketterle, Physical Review A 86, 021602 (2012)

6 C.-H. Wu, J. W. Park, P. Ahmadi, S. Will, and M. W. Zwierlein, Phys. Rev. Lett. 109, 085301 (2012)

7 T. A. Schulze, I. I. Temelkov, M. W. Gempel, T. Hartmann, H. Knöckel, S. Ospelkaus, and E. Tiemann, Physical Review A 88, 023401 (2013).

${ }^{8}$ S.-K. Tung, C. Parker, J. Johansen, C. Chin, Y. Wang, and P. S. Julienne, Physical Review A 87, 010702 (2013).

9 M. Repp, R. Pires, J. Ulmanis, R. Heck, E. D. Kuhnle, M. Weidemüller, and E. Tiemann, Physical Review A 87, 010701 (2013)

io T. Lahaye, C. Menotti, L. Santos, M. Lewenstein, and T. Pfau, Reports on Progress in Physics 72, 126401 (2009).

11 M. H. G. de Miranda, A. Chotia, B. Neyenhuis, D. Wang, G. Quemener, S. Ospelkaus, J. L. Bohn, J. Ye, and D. S. Jin, Nature Physics 7, 502 (2011).

12 A. Chotia, B. Neyenhuis, S. A. Moses, B. Yan, J. P. Covey, M. Foss-Feig, A. M. Rey, D. S. Jin, and J. Ye, Physical Review Letters 108, 080405 (2012)

is G. M. Bruun and E. Taylor, Physical Review Letters 101, 245301 (2008)

${ }^{14}$ N. R. Cooper and G. V. Shlyapnikov, Physical Review Letters 103, 155302 (2009).

is B. M. Fregoso, K. Sun, E. Fradkin, and B. L. Lev, New Journal of Physics 11, 103003 (2009)

to K. Sun, C. Wu, and S. Das Sarma, Physical Review B 82, 075105 (2010)

${ }^{17}$ Y. Yamaguchi, T. Sogo, T. Ito, and T. Miyakawa, Physical Review A 82, 013643 (2010)

18 N. T. Zinner and G. M. Bruun, The European Physical Journal D 65, 133 (2011)

19 M. Babadi and E. Demler, Physical Review A 84, 033636 (2011)

${ }^{20}$ L. M. Sieberer and M. A. Baranov, Physical Review A 84, 063633 (2011).

${ }^{21}$ M. M. Parish and F. M. Marchetti, Physical Review Letters 108, 145304 (2012).
${ }^{22}$ J. K. Block, N. T. Zinner, and G. M. Bruun, New Journal of Physics 14, 105006 (2012).

zs F. M. Marchetti and M. M. Parish, Physical Review B 87, 045110 (2013)

${ }^{24}$ G. M. Bruun and D. R. Nelson, Physical Review B 89, $094112(2014)$

${ }_{25}$ W. Lechner, H.-P. Büchler, and P. Zoller, ArXiv e-prints (2014), arXiv:1401.5682 [cond-mat.stat-mech].

26 S. A. Kivelson, E. Fradkin, and V. J. Emery, Nature 393, 550 (1998).

27 E. Fradkin and S. A. Kivelson, Science 327, 155 (2010).

28 N. Matveeva and S. Giorgini, Physical Review Letters 109, 200401 (2012)

${ }^{29}$ G. Baym and L. Kadanoff, Physical Review 124, 287 (1961)

so U. R. Fischer, Physical Review A 73, 031602 (2006).

31 T. Miyakawa, T. Sogo, and H. Pu, Physical Review A 77, 061603 (2008)

${ }^{32}$ M. Babadi and E. Demler, Physical Review B 84, 235124 (2011)

${ }_{3}$ P. M. Chaikin and T. C. Lubensky, Principles of Condensed Matter Physics (Cambridge University Press, 1995).

34 A. Andreev and I. Lifshitz, Sov.Phys.-JETP 29, 1107 (1969).

35 G. V. Chester, Physical Review A 2, 256 (1970).

36 A. J. Leggett, Physical Review Letters 25, 1543 (1970).

37 Y. E. Kim and A. L. Zubarev, Materials Science 70, 1 (2004)

38 M. H. W. Chan, Science 319, 1207 (2008)

39 B. Capogrosso-Sansone, C. Trefzger, M. Lewenstein, P. Zoller, and G. Pupillo, Physical Review Letters 104, 125301 (2010)

${ }^{40}$ M. Greiner, C. A. Regal, J. T. Stewart, and D. S. Jin, Physical Review Letters 94, 110401 (2005).

41 S. Fölling, F. Gerbier, A. Widera, O. Mandel, T. Gericke, and I. Bloch, Nature 434, 481 (2005).

42 T. Rom, T. Best, D. van Oosten, U. Schneider, S. Fölling, B. Paredes, and I. Bloch, Nature 444, 733 (2006).

${ }^{43}$ I. B. Spielman, W. D. Phillips, and J. V. Porto, Physical Review Letters 98, 080404 (2007)

44 T. Sogo, L. He, T. Miyakawa, S. Yi, H. Lu, and H. Pu, New Journal of Physics 11, 055017 (2009).

45 M. Babadi, B. Skinner, M. M. Fogler, and E. Demler, EPL (Europhysics Letters) 103, 16002 (2013)

${ }^{40}$ E. Fradkin, S. A. Kivelson, M. J. Lawler, J. P. Eisenstein, and A. P. Mackenzie, Annual Review of Condensed Matter Physics 1, 153 (2010).

4 V. L. Berezinskii, Sov. Phys. JETP 34, 610 (1972).

48 J. M. Kosterlitz and D. J. Thouless, Journal of Physics C: Solid State Physics 6, 1181 (1973) 